iting cardiac hypertrophy (1), other loss-offunction approaches appear ineffective. We remain enthusiastic about miRNAs as therapeutic targets and welcome such dialog.

\section{David M. Patrick, ${ }^{1}$ Sakari Kauppinen, ${ }^{2}$ Eva van Rooij, ${ }^{3}$ and Eric N. Olson'1}

${ }^{1}$ Department of Molecular Biology, University of Texas Southwestern Medical Center, Dallas, Texas, USA. ${ }^{2}$ Santaris Pharma, Hørsholm, Denmark. ${ }^{3}$ miRagen Therapeutics,
Boulder, Colorado, USA.

Conflict of interest: Eric N. Olson and Eva van Rooij are co-founders of miRagen Therapeutics. Sakari Kauppinen is employed at Santaris Pharma.

Address correspondence to: Eric Olson, University of Texas Southwestern Medical Center, 6000 Harry Hines Blvd., Dallas, Texas 75390-9148, USA. Phone: 214.648.1187; Fax: 214.648.1196; E-mail: Eric.Olson@ utsouthwestern.edu.
J Clin Invest. 2011;121(2):462-463. doi:10.1172/JCI46108.

1. Thum T, et al. MicroRNA-21 contributes to myocardial disease by stimulating MAP kinase signaling in fibroblasts. Nature. 2008;456(7224):980-984.

2. Patrick DM, et al. Stress-dependent cardiac remodeling occurs in the absence of miR-21 in mice. JClin Invest. 2010;120(11):3912-3916.

3. Hatley ME, et al. Modulation of K-Ras-dependent lung tumorigenesis by microRNA-21. Cancer Cell. 2010;18(3):282-293.

4. van Rooij E, Sutherland LB, Qi X, Richardson JA, Hill J, Olson EN. Control of stress-dependent cardiac growth and gene expression by a microRNA. Science. 2007;316(5824):575-579.

\title{
Expression of TNFRSF25 on conventional T cells and Tregs
}

mmunological tolerance is achieved through recessive and dominant mechanisms. In recessive tolerance the fate of selfreactive $T$ cells is controlled in a cell intrinsic manner such that they undergo cell death or become anergic after exposure to selfantigen. In contrast, dominant tolerance is cell extrinsic and is mediated by $\mathrm{CD}^{+}$ Tregs that express the transcription factor Forkhead P3 (Foxp3) and show increased self-reactivity when compared with conventional T cells (1). Peripheral homeostasis of Tregs is maintained through $\mathrm{T}$ cell receptor stimulation and signaling by IL- 2 and costimulatory receptors such as CD28 $(2,3)$. Identifying the full spectrum of signals controlling Treg homeostasis in vivo may lend itself to therapeutic strategies that can be used to manipulate their number or function in order to ameliorate inflammatory diseases or augment an antitumor immune response. In this regard, the recent study by Schreiber and colleagues in the JCI (4) is particularly interesting, since the authors demonstrate that injection of mice with a mAb that binds to TNF receptor superfamily member 25 (TNFRSF25) selectively expands Tregs. The authors also show that administration of the anti-TNFRSF $25 \mathrm{mAb}$ inhibits a Th2 inflammatory response in the lungs of antigen-sensitized mice. Another interesting observation reported in that study was the preferential binding of the anti-TNFRSF25 mAb to Tregs (4). Based on this, the authors concluded that the level of TNFRSF 25 on Tregs is considerably higher than that found on conventional $\mathrm{CD}^{+}$ $\mathrm{T}$ cells. We have reexamined the expression of TNFRSF 25 on conventional CD $4^{+} \mathrm{T}$ cells and Tregs, and our findings are remarkably different to those reported by Schreiber et al. We used two approaches to investigate expression of TNFRSF25 on T cell subsets (Figure 1). First, we showed that a different anti-TNFRSF25 antibody stains conventional $\mathrm{T}$ cells and Tregs obtained from Foxp3GFP knockin mice (5) with similar intensity (Figure 1, A-C). Second, we demonstrated that soluble recombinant TL1A (sTL1A), the ligand for TNFRSF25, binds equally well to conventional $\mathrm{CD} 4^{+} \mathrm{T}$ cells and Tregs (Figure 1D). We therefore conclude that Tregs and conventional $\mathrm{CD}^{+} \mathrm{T}$ cells express similar levels of functional TNFRSF25.

We agree with the findings of Schreiber et al. that TNFRSF25 triggering can expand Tregs. In fact, we recently showed that transgenic mice that constitutively express TL1A have increased numbers of Tregs (6). However, we also observed increased activation of conventional $\mathrm{CD}^{+} \mathrm{T}$ cells, elevated levels of IL-13 and IL-17, and small intestinal immune pathology that manifests as goblet cell and paneth cell hyperplasia (6). A similar phenotype was reported by Siegel and colleagues using mice that express higher levels of a TL1A transgene (7). Interestingly, we found that stimulation of $\mathrm{T}$ cells with recombinant TL1A attenuates Treg-mediated suppression in vitro and this effect required TNFRSF25 signaling in either conventional CD4 ${ }^{+} \mathrm{T}$ cells or Tregs (6). Thus, TNFRSF25 exerts costimulatory effects on conventional CD $4^{+} \mathrm{T}$ cells as well as on Tregs. Furthermore, its effects on promoting Treg turnover are counterbalanced by its ability to attenuate immune suppression and stimulate effector $\mathrm{T}$ cells. This conclusion is in line with findings obtained using mice that were rendered deficient in either TNFRSF25 or TL1A. In these mice, the net effect of defective TNFRSF25 signaling is a reduction in the severity of T cell-mediated inflammation $(8,9)$.

How then can these findings been reconciled? We suspect that the anti-TNFRSF25 $\mathrm{mAb}$ used by Schreiber et al. may bind to an epitope that is preferentially expressed on Tregs, since multiple mRNA isoforms of TNFRSF25 have been described (10), and this may have lead to preferential expansion of Tregs in their experiments. Indeed, this notion is supported by a previous study showing differences in TNFRSF25 mRNA isoform expression between Tregs and Th17 cells (8). Thus, when compared with conventional T cells, Tregs expressed more TNFRSF25 mRNA transcripts that encode the shorter variant of TNFRSF25, which lacks the fourth extracellular cysteine-rich repeat. It is noteworthy that the antibody used in our study bound to both full-length TNFRSF25 as well as the short variant lacking the fourth cysteine-rich repeat (Figure 1E). We believe that caution should be applied in interpreting the findings obtained with anti-TNFRSF25 antibodies, as these may differ in their capacity to stimulate Tregs and effector $T$ cells.

\section{Acknowledgments}

This work is funded by Leukaemia \& Lymphoma Research.

\section{Vadim Y. Taraban, John R. Ferdinand, and Aymen Al-Shamkhani}


A

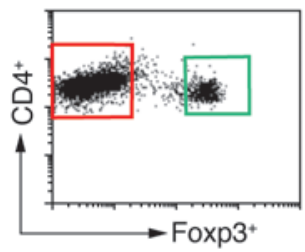

C

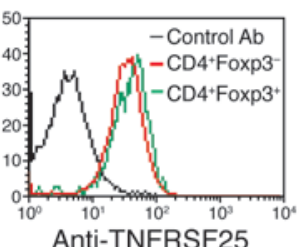

E

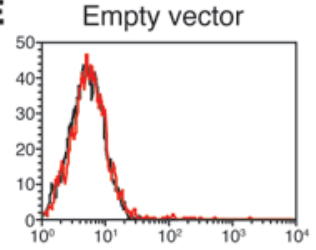

B Nonpermeabilized cells
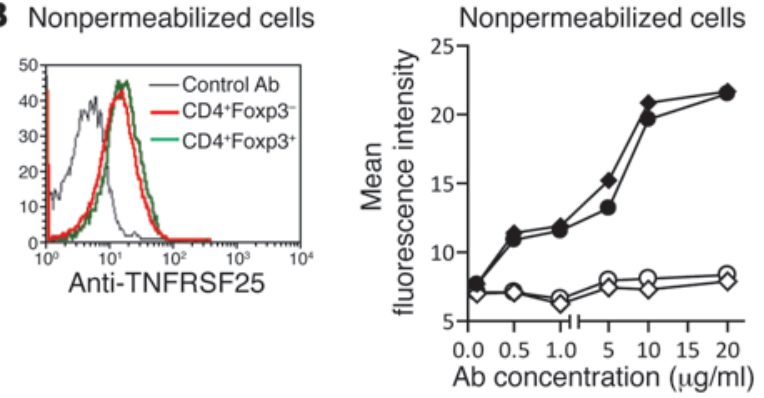

D
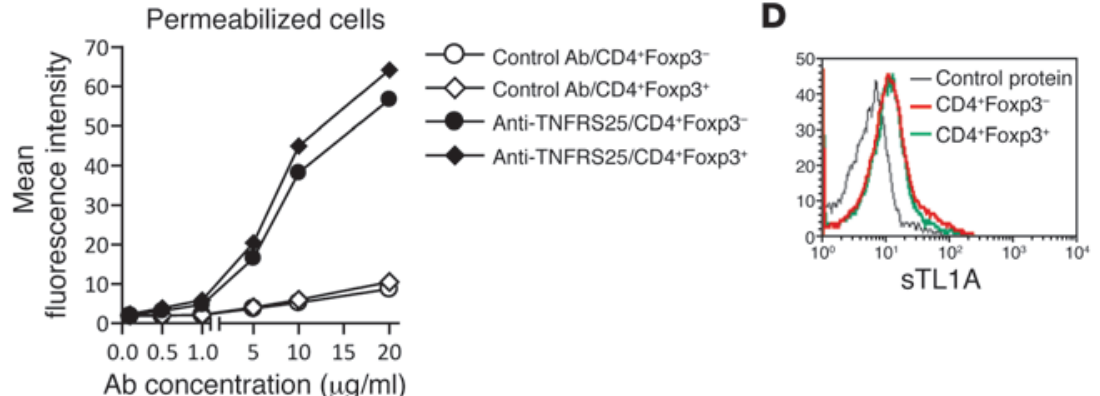

$-\mathrm{O}-$ Control Ab/CD4 ${ }^{+} \mathrm{Foxp3}^{-}$ $\checkmark$ Control Ab/CD4 ${ }^{+}$Foxp3 $^{+}$

- - Anti-TNFRS25/CD4 ${ }^{*}$ Foxp3Anti-TNFRS25/CD4*Foxp3*
Full-length TNFRSF25

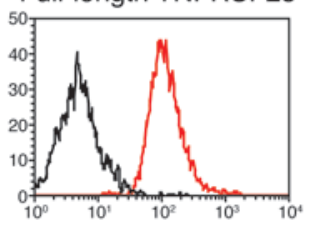

Short TNFRSF25 variant

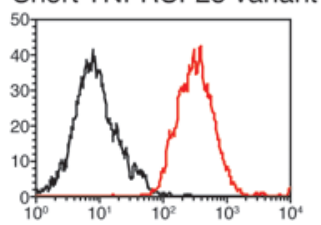

\section{Figure 1}

CD4 ${ }^{+}$conventional T cells and Tregs express similar levels of TNFRSF25. (A) A flow cytometric dot plot showing the gating of CD4 ${ }^{+}$Foxp3- and CD4+Foxp3+ T cells. (B-D) Binding of anti-TNFRSF25 antibody (B and C) or STL1A (D) to CD4+Foxp3- and CD4+Foxp3 ${ }^{+}$T cells. Splenocytes from Foxp3-GFP knockin mice were incubated with anti-FcyRII/III (2.4G2) and then phycoerythrin-conjugated anti-CD4 mAbs. After washing, cells were incubated with affinity-purified biotinylated goat anti-mouse TNFRSF25 IgG (R\&D Systems) or normal biotinylated goat IgG for 40 minutes at $4{ }^{\circ} \mathrm{C}$. Binding was detected using allophycocyanin-conjugated streptavidin. Histograms are electronically gated on CD4+GFP- or CD4 ${ }^{+}$GFP $^{+}$cells. To detect TNFRSF25 intracellularly, cells were permeabilized using Cytofix/Cytoperm solution (BD Biosciences). For staining with sTL1A, cells were first incubated with anti-Fc $\gamma$ RII/III and then phycoerythrin-conjugated anti-CD4 mAbs. After washing, cells were incubated $\left(40\right.$ minutes, $\left.4^{\circ} \mathrm{C}\right)$ with affinity-purified STL1A, which consists of an N-terminal, rat CD4 domains 3 and 4 tag, and the extracellular domain of mouse TL1A (6), or with bovine serum albumin as a control. Binding was detected using allophycocyanin-conjugated mouse anti-tag mAb (OX68). Histograms in B-D are representative of 4 independent experiments in which anti-TNFRSF25 antibody or sTL1A were used at $10 \mu \mathrm{g} / \mathrm{ml}$. (E) Anti-TNFRSF25 antibody binds to full-length TNFRSF25 and the short variant lacking the fourth extracellular cysteine-rich repeat. NIH3T3 cells transduced with an "empty" recombinant retrovirus or recombinant virus that encodes full-length TNFRSF25 or the short TNFRSF25 variant were stained with anti-TNFRSF25 or control antibody as described above.

Cancer Sciences Division, University of Southampton School of Medicine, Southampton, United Kingdom.

Conflict of interest: The authors have declared that no conflict of interest exists.

Address correspondence to: A. Al-Shamkhani, Cancer Sciences Division, University of Southampton School of Medicine, Tenovus Research Laboratory, Southampton General Hospital, Tremona Road, Southampton, SO16 6YD, United Kingdom. Phone: 44.23.8079.6285; Fax: 44.23.8070.4061; E-mail: aymen@soton.ac.uk.
J Clin Invest. 2011;121(2):463-464. doi:10.1172/JCI45832.

1. Sakaguchi S, Yamaguchi T, Nomura T, Ono M. Regulatory $\mathrm{T}$ cells and immune tolerance. Cell. 2008;133(5):775-787.

2. Josefowicz SZ, Rudensky A. Control of regulatory T cell lineage commitment and maintenance. Immunity. 2009;30(5):616-625.

3. Bour-Jordan H, Bluestone JA. Regulating the regulators: costimulatory signals control the homeostasis and function of regulatory $\mathrm{T}$ cells. Immunol Rev. 2009;229(1):41-66.

4. Schreiber TH, et al. Therapeutic Treg expansion in mice by TNFRSF 25 prevents allergic lung inflammation. J Clin Invest. 2010;120(10):3629-3640.

5. Bettelli E, et al. Reciprocal developmental pathways for the generation of pathogenic effector TH17 and regulatory T cells. Nature. 2006;441(7090):235-238.
6. Taraban VY, et al. Sustained TL1A expression modulates effector and regulatory T-cell responses and drives intestinal goblet cell hyperplasia [published online ahead of print October 20, 2010]. Mucosal Immunol. doi:10.1038/mi.2010.70.

7. Meylan F, et al. The TNF-family cytokine TL1A drives IL-13-dependent small intestinal inflammation [published online ahead of print October 27, 2010]. Mucosal Immunol. doi:10.1038/mi.2010.67.

8. Pappu BP, et al. TL1A-DR3 interaction regulates Th17 cell function and Th17-mediated autoimmune disease. J Exp Med. 2008;205(5):1049-1062.

9. Meylan F, et al. The TNF-family receptor DR3 is essential for diverse $\mathrm{T}$ cell-mediated inflammatory diseases. Immunity. 2008;29(1):79-89.

10. Wang EC, Kitson J, Thern A, Williamson J, Farrow $\mathrm{SN}$, Owen MJ. Genomic structure, expression, and chromosome mapping of the mouse homologue for the WSL-1 (DR3, Apo3, TRAMP, LARD, TR3, TNFRSF12) gene. Immunogenetics. 2001;53(1):59-63. 